\title{
]jfis
}

\section{On Separation Axioms in Fuzzifying Bitopological Spaces}

\author{
Ahmed Abd El-Monsef Allam ${ }^{1}$, Ahmed Mohammed Zahran² ${ }^{2}$, Ahmed Khalf \\ Mousa $^{2,3}$, and Hana Mohsen Binshahnah ${ }^{4}$ \\ ${ }^{1}$ Department of Mathematics, Faculty of Science, Assiut University, Assiut, Egypt \\ ${ }^{2}$ Department of Mathematics, Faculty of Science, Al-Azhar University, Assiut, Egypt \\ ${ }^{3}$ Department of Mathematics, University of Tabuk at Al-Wajh, Saudi Arabia \\ ${ }^{4}$ Department of Mathematics, Faculty of Science, Hadhramout University, Republic of Yemen
}

\begin{abstract}
In present paper, we introduce and study the concepts of $T_{0}^{(i, j)}-, T_{1}^{(i, j)}-, T_{2}^{(i, j)}$ (pairwise Hausdorff)-, $T_{R}^{(i, j)}$ (pairwise regularity)-, $T_{N}^{(i, j)}$ (pairwise normality)-, $R_{0}^{(i, j)}-, R_{1}^{(i, j)}-, T_{3}^{(i, j)}$ and $T_{4}^{(i, j)}$ - separation axioms in fuzzifying bitopological spaces and study some relations between them. Also we investigate the image of these kinds of fuzzifying bitopological spaces under some types of fuzzy mappings.
\end{abstract}

Keywords: Fuzzifying topology, Fuzzifying bitopological space, Separation axioms

\section{Introduction}

In 1963, Kelley [1] introduced the notions of bitopological spaces. Such spaces equipped with its two (arbitrary) topologies. In 1991-1993, Ying [2-4] introduced the concept of the fuzzifying topology with the sematic method of continuous valued logic. On the framework of fuzzifying topology, Shen [5] introduced and studied the notions of separation axioms. In 2003, Zhang and Liu [6] studied the concepts of fuzzy $\theta_{(i, j)}$-closed, $\theta_{(i, j)}$-open sets in fuzzifying bitopological spaces. In 2007, Kiliciman and Salleh [7] introduced two concepts of pairwise Landelöf bitopological spaces and studied the properties of them.

The contains of this paper are arranged as follows. In Section 3, we introduce $T_{0}^{(i, j)}$-, $T_{1}^{(i, j)}-, T_{2}^{(i, j)}$ (pairwise Hausdorff)-, $T_{R}^{(i, j)}$ (regularity)-, $T_{N}^{(i, j)}$ (pairwise normality)-, $R_{0}^{(i, j)}$ and $R_{1}^{(i, j)}$ - separation axioms in fuzzifying bitopological spaces. We define $T_{3}^{(i, j)}, T_{4}^{(i, j)}$ and new weaker form of the pairwise normality axioms in fuzzifying bitopological spaces. In Section 4, we study some important relations between that separation axioms in fuzzifying bitopological spaces. Finally in Section 5, we investigate the image of these kinds of fuzzifying bitopological spaces under some types of fuzzy mappings.

(c)This is an Open Access article distributed under the terms of the Creative Commons Attribution Non-Commercial License (http://creativecommons.org/licenses/ by-nc/3.0// which permits unrestricted noncommercial use, distribution, and reproduction in any medium, provided the original work is properly cited.

\section{Preliminaries}

Firstly, we display the fuzzy logical and corresponding set-theoretical notations used in this paper. For formula $\varphi$, the symbol $[\varphi]$ means the truth of $\varphi$, where the set of truth values is the unit interval $[0,1]$. A formula $\varphi$ is valid, we write $\models \varphi$ if and only if $[\varphi]=1$ for every interpretation. 
(1) $[\alpha]:=\alpha(\alpha \in[0,1]) ;[\alpha \wedge \beta]=\min ([\alpha],[\beta]) ;[\alpha \rightarrow$ $\beta]=\min (1,1-[\alpha]+[\beta])$,

$[\forall x \alpha(x)]=\inf _{x \in X}[\alpha(x)]$,

where $X$ is the universe of discourse.

(2) If $\widetilde{A} \in \Im(X)$, where $\Im(X)$ is the family of fuzzy sets of $X$, then $[x \in \widetilde{A}]:=\widetilde{A}(x)$.

(3) If $X$ is the universe of discourse, then $[\forall x \alpha(x)]=$ $\inf _{x \in X}[\alpha(x)]$.

In addition, the following derived formulae are given:

(1) $[\neg \alpha]=1-[\alpha]$.

(2) $[\alpha \vee \beta]=\max ([\alpha],[\beta])$.

(3) $[\alpha \leftrightarrow \beta]:=[(\alpha \rightarrow \beta) \wedge(\beta \rightarrow \alpha)]$.

(4) $[\alpha \dot{\wedge} \beta]=\max (0,[\alpha]+[\beta]-1)$.

(5) $[\alpha \dot{\vee} \beta]=\min (1,[\alpha]+[\beta])$.

(6) $[\exists x \alpha(x)]:=[\neg(\forall x \neg \alpha(x))]$.

(7) If $\widetilde{A}, \widetilde{B} \in \Im(X)$, then

(a) $[\widetilde{A} \subseteq \widetilde{B}]:=[\forall x(x \in \widetilde{A} \rightarrow x \in \widetilde{B})]=\inf _{x \in X} \min (1,1-$ $\widetilde{A}(x)+\widetilde{B}(x))$,

(b) $[A \equiv B]:=[(\widetilde{A} \subseteq \widetilde{B}) \wedge(\widetilde{B} \subseteq \widetilde{A})]$.

Secondly, we give the following definitions which are used in the sequel.

Definition 2.1 ([2]). Let $X$ be a universe of discourse and $\tau \in \Im(P(X))$ satisfy the following conditions:

(1) $\tau(X)=1$ and $\tau(\phi)=1$;

(2) for any $A, B, \tau(A \cap B) \geq \tau(A) \wedge \tau(B)$;

(3) for any $\left\{A_{\lambda}: \lambda \in \Lambda\right\}, \tau\left(\bigcup_{\lambda \in \Lambda} A_{\lambda}\right) \geq \bigwedge_{\lambda \in \Lambda} \tau\left(A_{\lambda}\right)$.

Then $\tau$ is a fuzzifying topology and $(X, \tau)$ a fuzzifying topological space.

Definition $2.2([2])$. Let $(X, \tau)$ be a fuzzifying topological space. Then

(1) The family of all fuzzifying closed sets is denoted by $\mathcal{F} \in \Im(P(X))$, and defined as follows: $A \in \mathcal{F}:=X \sim A \in \tau$, where $X \sim A$ is the complement of $A$.

(2) The neighborhood system of $x \in X$ is denoted by $N_{x} \in$ $\Im(P(X))$ and defined as follows: $N_{x}(A)=\sup _{x \in B \subseteq A} \tau(B)$.

(3) The closure $\operatorname{cl}(A)$ of $A \subseteq X$ is defined as follows: $\operatorname{cl}(A)(x)=1-N_{x}(X \sim A)$.
Definition 2.3 ([4]]). Let $(X, \tau)$ and $(Y, \sigma)$ be two fuzzifying topological spaces.

(1) A unary fuzzy predicate $C \in \Im\left(Y^{X}\right)$, called fuzzy continuity, is given as follows: $f \in C:=\forall u\left(u \in \sigma \rightarrow f^{-1}(u) \in\right.$ $\tau)$. i.e., $C(f)=\inf _{u \in P(Y)} \min \left(1,1-\sigma(u)+\tau\left(f^{-1}(u)\right)\right)$.

(2) A unary fuzzy predicate $O \in \Im\left(Y^{X}\right)$, called fuzzy openness, is given as follows: $f \in O:=\forall u(u \in \tau \rightarrow f(u) \in \sigma)$. i.e, $O(f)=\inf _{u \in P(X)} \min (1,1-\tau(u)+\sigma(f(u)))$.

Definition $2.4([\mid 6])$. Let $\left(X, \tau_{1}\right)$ and $\left(X, \tau_{2}\right)$ be two fuzzifying topological spaces. Then a system $\left(X, \tau_{1}, \tau_{2}\right)$ consisting of a universe of discourse $X$ with two fuzzifying topologies $\tau_{1}$ and $\tau_{2}$ on $X$ is called a fuzzifying bitopological space.

Definition 2.5 ( [8]). Let $\left(X, \tau_{1}, \tau_{2}\right)$ and $\left(Y, \sigma_{1}, \sigma_{2}\right)$ be two fuzzifying bitopological spaces. A mapping $f:\left(X, \tau_{1}, \tau_{2}\right) \rightarrow$ $\left(Y, \sigma_{1}, \sigma_{2}\right)$ is said to be pairwise fuzzy continuous (resp. pairwise fuzzy open) if $f:\left(X, \tau_{1}\right) \rightarrow\left(Y, \sigma_{1}\right)$ and $f:\left(X, \tau_{2}\right) \rightarrow$ $\left(Y, \sigma_{2}\right)$ are fuzzy continuous (resp. fuzzy open).

Lemma 2.1 ([9]). For any $\alpha, \beta \in[0,1], \models \alpha \rightarrow(\beta \rightarrow \alpha)$.

\section{Separation Axioms in Fuzzifying Bitopologi- cal Spaces}

Definition 3.1. Let $\left(X, \tau_{1}, \tau_{2}\right)$ be a fuzzifying bitopological space.

(1) A set $A$ is said to be a pairwise open if and only if $A \in \tau_{1} \cap \tau_{2}$, i.e.,

$$
\mathcal{O}^{p}(A)=\min \left(\tau_{1}(A), \tau_{2}(A)\right)
$$

(2) A set $B$ is said to be a pairwise closed if and only if $X \sim B \in \mathcal{O}^{p}$, i.e.,

$$
B \in \mathcal{F}^{p}:=X \sim B \in \mathcal{O}^{p} .
$$

Lemma 3.1. Let $\left(X, \tau_{1}, \tau_{2}\right)$ be a fuzzifying bitopological space. Then

$$
\mathcal{F}^{p}(B)=\min \left(\mathcal{F}_{1}(B), \mathcal{F}_{2}(B)\right) .
$$

Proof. $\mathcal{F}^{p}(B)=\mathcal{O}^{p}(X \sim B)=\min \left(\tau_{1}(X \sim B), \tau_{2}(X \sim\right.$ $B))=\min \left(\mathcal{F}_{1}(B), \mathcal{F}_{2}(B)\right)$.

Lemma 3.2. Let $\left(X, \tau_{1}, \tau_{2}\right)$ be a fuzzifying bitopological space. Then
(1) $\models \mathcal{O}^{p} \subseteq \tau_{i}, i=1,2$.
(2) $\models \mathcal{F}^{p} \subseteq \mathcal{F}_{i}, i=1,2$. 
Proof.

(1) $\mathcal{O}^{p}(A)=\min \left(\tau_{1}(A), \tau_{2}(A)\right) \leq \tau_{i}(A)$.

(2) It is similar to (1) above.

Remark 3.1. For simplicity we put the following notations:

$$
K_{x, y}^{(i, j)}:=\exists A\left(\left(A \in N_{x}^{i} \wedge y \notin A\right) \vee\left(A \in N_{y}^{j} \wedge x \notin A\right)\right),
$$

where $N_{x}^{i}$ is the neighborhood system of $x$ with respect to $\tau_{i}$,

$$
\begin{aligned}
H_{x, y}^{(i, j)}:= & \exists B \exists C\left(B \in N_{x}^{i} \wedge C \in N_{y}^{j} \wedge y \notin B \wedge x \notin C\right), \\
M_{x, y}^{(i, j)}:= & \exists \exists C\left(B \in N_{x}^{i} \wedge C \in N_{y}^{j} \wedge B \cap C=\phi\right), \\
W_{A, B}^{(i, j)}:= & \exists U \exists V\left(U \in \tau_{i} \wedge V \in \tau_{j} \wedge A \subseteq V \wedge B\right. \\
& \subseteq U \wedge U \cap V=\phi) .
\end{aligned}
$$

Definition 3.2. Let $\Omega$ be the class of all fuzzifying bitopological spaces. The unary fuzzy predicates $T_{n}^{(i, j)} \in \Im(\Omega), n=0,1$, $2, T_{N}^{(i, j)} \in \Im(\Omega)$ and $R_{n}^{(i, j)} \in \Im(\Omega), n=0,1$ are defined as follows respectively:

$$
\begin{aligned}
& \left(X, \tau_{1}, \tau_{2}\right) \in T_{0}^{(i, j)} \\
& :=\forall x \forall y\left(x \in X \wedge y \in X \wedge x \neq y \rightarrow K_{x, y}^{(i, j)}\right), \\
& \left(X, \tau_{1}, \tau_{2}\right) \in T_{1}^{(i, j)} \\
& :=\forall x \forall y\left(x \in X \wedge y \in X \wedge x \neq y \rightarrow H_{x, y}^{(i, j)}\right), \\
& \left(X, \tau_{1}, \tau_{2}\right) \in T_{2}^{(i, j)} \\
& :=\forall x \forall y\left(x \in X \wedge y \in X \wedge x \neq y \rightarrow M_{x, y}^{(i, j)}\right), \\
& \left(X, \tau_{1}, \tau_{2}\right) \in T_{N}^{(i, j)} \\
& :=\forall A \forall B\left(A \in \mathcal{F}_{i} \wedge B \in \mathcal{F}_{j} \wedge A \cap B=\phi \rightarrow W_{A, B}^{(i, j)}\right), \\
& \left(X, \tau_{1}, \tau_{2}\right) \in R_{0}^{(i, j)} \\
& :=\forall x \forall y\left(x \in X \wedge y \in X \wedge x \neq y \rightarrow\left(K_{x, y}^{(i, j)} \rightarrow H_{x, y}^{(i, j)}\right)\right), \\
& \left(X, \tau_{1}, \tau_{2}\right) \in R_{1}^{(i, j)} \\
& :=\forall x \forall y\left(x \in X \wedge y \in X \wedge x \neq y \rightarrow\left(K_{x, y}^{(i, j)} \rightarrow M_{x, y}^{(i, j)}\right)\right) .
\end{aligned}
$$

Remark 3.2. Let $\left(X, \tau_{1}, \tau_{2}\right)$ be a fuzzifying bitopological space. Note that

(1) $T_{n}^{(1,2)}=T_{n}^{(2,1)}, n=0,1,2$.

(2) $T_{N}^{(1,2)}=T_{N}^{(2,1)}$.

(3) $R_{n}^{(1,2)}=R_{n}^{(2,1)}, n=0,1$.

Definition 3.3. Let $\Omega$ be the class of all fuzzifying bitopological spaces. The unary fuzzy predicates $T_{R_{i}}^{(i, j)}, T_{R_{j}}^{(i, j)}$ and $T_{R}^{(i, j)} \in \Im(\Omega)$, are defined as follows:
(1) $\left(X, \tau_{1}, \tau_{2}\right) \in T_{R_{i}}^{(i, j)}:=\forall x \forall U\left(x \in X \wedge U \in \mathcal{F}_{i} \wedge x \notin\right.$ $\left.U \rightarrow \exists A \exists B\left(A \in N_{x}^{i} \wedge B \in \tau_{j} \wedge U \subseteq B \wedge A \cap B=\phi\right)\right)$.

(2) $\left(X, \tau_{1}, \tau_{2}\right) \in T_{R_{j}}^{(i, j)}:=\forall x \forall U\left(x \in X \wedge U \in \mathcal{F}_{j} \wedge x \notin\right.$ $\left.U \rightarrow \exists A \exists B\left(A \in N_{x}^{j} \wedge B \in \tau_{i} \wedge U \subseteq B \wedge A \cap B=\phi\right)\right)$.

(3) $\left(X, \tau_{1}, \tau_{2}\right) \in T_{R}^{(i, j)}:=\left(X, \tau_{1}, \tau_{2}\right) \in T_{R_{i}}^{(i, j)} \wedge\left(X, \tau_{1}, \tau_{2}\right)$ $\in T_{R_{j}}^{(i, j)}$.

The following example shows that generally $T_{R_{i}}^{(i, j)}=T_{R_{j}}^{(i, j)}$ need not be true.

Example 3.1. Let $X=\{a, b, c\}$ and $\tau_{1}, \tau_{2}$ be two fuzzifying topologies defined as follows:

$$
\begin{aligned}
& \tau_{1}(A)= \begin{cases}1, & \text { if } A \in\{\phi, X,\{a, b\}\}, \\
1 / 4, & \text { if } A=\{c\}, \\
0, & \text { if } A \in\{\{a\},\{b\},\{a, c\},\{b, c\}\},\end{cases} \\
& \tau_{2}(A)= \begin{cases}1, & \text { if } A \in\{\phi, X,\{a, b\}\}, \\
1 / 8, & \text { if } A=\{c\}, \\
0, & \text { textif } A \in\{\{a\},\{b\},\{a, c\},\{b, c\}\} .\end{cases}
\end{aligned}
$$

Note that

$$
\begin{aligned}
& \mathcal{F}_{1}(A)= \begin{cases}1, & \text { if } A \in\{\phi, X,\{c\}\}, \\
1 / 4, & \text { if } A=\{a, b\}, \\
0, & \text { if } A \in\{\{b, c\},\{a, c\},\{b\},\{a\}\},\end{cases} \\
& \mathcal{F}_{2}(A)= \begin{cases}1, & \text { if } A \in\{\phi, X,\{c\}\}, \\
1 / 8, & \text { if } A=\{a, b\}, \\
0, & \text { if } A \in\{\{b, c\},\{a, c\},\{b\},\{a\}\} .\end{cases}
\end{aligned}
$$

Then we have $T_{R_{1}}^{(1,2)}\left(X, \tau_{1}, \tau_{2}\right)=1 / 8 \neq 1 / 4=T_{R_{2}}^{(1,2)}\left(X, \tau_{1}\right.$, $\left.\tau_{2}\right)$.

Definition 3.4. Let $\Omega$ be the class of all fuzzifying bitopological spaces. The unary fuzzy predicates $T_{3}^{(i, j)}, T_{4}^{(i, j)} \in \Im(\Omega)$ are defined as follows respectively:

(1) $T_{3}^{(i, j)}\left(X, \tau_{1}, \tau_{2}\right):=T_{R}^{(i, j)}\left(X, \tau_{1}, \tau_{2}\right) \dot{\wedge} T_{1}^{(i, j)}\left(X, \tau_{1}, \tau_{2}\right)$.

(2) $T_{4}^{(i, j)}\left(X, \tau_{1}, \tau_{2}\right):=T_{N}^{(i, j)}\left(X, \tau_{1}, \tau_{2}\right) \dot{\wedge} T_{1}^{(i, j)}\left(X, \tau_{1}, \tau_{2}\right)$.

Lemma 3.3. Let $\left(X, \tau_{1}, \tau_{2}\right)$ be a fuzzifying bitopological space. Then
(1) $\models M_{x, y}^{(i, j)} \rightarrow H_{x, y}^{(i, j)}$.
(2) $\models H_{x, y}^{(i, j)} \rightarrow K_{x, y}^{(i, j)}$.
(3) $\models M_{x, y}^{(i, j)} \rightarrow K_{x, y}^{(i, j)}$.

Proof. The proof is obvious. 
Theorem 3.1. Let $\left(X, \tau_{1}, \tau_{2}\right)$ be a fuzzifying bitopological space. Then
$(1) \models\left(X, \tau_{1}, \tau_{2}\right) \in T_{1}^{(i, j)} \rightarrow\left(X, \tau_{1}, \tau_{2}\right) \in T_{0}^{(i, j)}$.
$(2) \models\left(X, \tau_{1}, \tau_{2}\right) \in T_{2}^{(i, j)} \rightarrow\left(X, \tau_{1}, \tau_{2}\right) \in T_{1}^{(i, j)}$.
(3) $\models\left(X, \tau_{1}, \tau_{2}\right) \in T_{2}^{(i, j)} \rightarrow\left(X, \tau_{1}, \tau_{2}\right) \in T_{0}^{(i, j)}$.

Proof. From Lemma 3.3 it is clear.

Theorem 3.2. Let $\left(X, \tau_{1}, \tau_{2}\right)$ be a fuzzifying bitopological space. Then

$$
\begin{aligned}
& (1) \models\left(X, \tau_{1}, \tau_{2}\right) \in R_{1}^{(i, j)} \rightarrow\left(X, \tau_{1}, \tau_{2}\right) \in R_{0}^{(i, j)} . \\
& (2) \models\left(X, \tau_{1}, \tau_{2}\right) \in T_{1}^{(i, j)} \rightarrow\left(X, \tau_{1}, \tau_{2}\right) \in R_{0}^{(i, j)} . \\
& (3) \models\left(X, \tau_{1}, \tau_{2}\right) \in T_{2}^{(i, j)} \rightarrow\left(X, \tau_{1}, \tau_{2}\right) \in R_{0}^{(i, j)} . \\
& (4) \models\left(X, \tau_{1}, \tau_{2}\right) \in T_{2}^{(i, j)} \rightarrow\left(X, \tau_{1}, \tau_{2}\right) \in R_{1}^{(i, j)} .
\end{aligned}
$$

Proof. From Lemma 2.1. Lemma 3.3 and Theorem 3.1. the proof becomes obvious.

Theorem 3.3. Let $\left(X, \tau_{1}, \tau_{2}\right)$ be a fuzzifying bitopological space. Then

$$
\models\left(X, \tau_{1}, \tau_{2}\right) \in T_{0}^{(i, j)} \leftrightarrow \forall x \forall y(x \in X \wedge y \in X \wedge x \neq
$$
$\left.y \rightarrow x \notin \operatorname{cl}_{i}(\{y\}) \vee y \notin \operatorname{cl}_{j}(\{x\})\right)$.

Proof. The proof is obvious.

Theorem 3.4. Let $\left(X, \tau_{1}, \tau_{2}\right)$ be a fuzzifying bitopological space. Then

$$
\begin{aligned}
& (1) \models\left(X, \tau_{1}, \tau_{2}\right) \in T_{1}^{(i, j)} \rightarrow \forall x\left(\{x\} \in \mathcal{F}_{i}\right) . \\
& (2) \models\left(X, \tau_{1}, \tau_{2}\right) \in T_{1}^{(i, j)} \rightarrow \forall x\left(\{x\} \in \mathcal{F}_{j}\right) .
\end{aligned}
$$

Proof.

$$
\text { (1) } \begin{aligned}
& T_{1}^{(i, j)}\left(X, \tau_{1}, \tau_{2}\right) \\
& =\inf _{x_{1} \neq x_{2}} \min \left(\sup _{x_{2} \notin A} N_{x_{1}}^{i}(A), \sup _{x_{1} \notin B} N_{x_{2}}^{j}(B)\right) \\
& =\inf _{x_{1} \neq x_{2}} \min \left(N_{x_{1}}^{i}\left(X \sim\left\{x_{2}\right\}\right), N_{x_{2}}^{j}\left(X \sim\left\{x_{1}\right\}\right)\right) \\
& \leq \inf _{x_{1} \neq x_{2}} N_{x_{1}}^{i}\left(X \sim\left\{x_{2}\right\}\right) \\
& =\inf _{x_{2} \in X} \inf _{x_{1} \in X \sim\left\{x_{2}\right\}} N_{x_{1}}^{i}\left(X \sim\left\{x_{2}\right\}\right) \\
& =\inf _{x_{2} \in X} \tau_{i}\left(X \sim\left\{x_{2}\right\}\right) \\
& =\inf _{x \in X} \tau_{i}(X \sim\{x\})=\inf _{x \in X} \mathcal{F}_{i}(\{x\}) .
\end{aligned}
$$

(2) It is similar to (1) above.

The following examples show that generally the reverse of Theorem 3.4 need not be true.
Example 3.2. Let $X=\{a, b\}$ and $\tau_{1}, \tau_{2}$ be two fuzzifying topologies defined as follows:

$$
\begin{gathered}
\tau_{1}(A)= \begin{cases}1, & \text { if } A \in\{\phi, X\}, \\
1 / 5, & \text { if } A=\{a\}, \\
1 / 2, & \text { if } A=\{b\},\end{cases} \\
\tau_{2}(A)= \begin{cases}1, & \text { if } A \in\{\phi, X\}, \\
1 / 4, & \text { if } A=\{a\}, \\
1 / 8, & \text { if } A=\{b\} .\end{cases}
\end{gathered}
$$

Note that

$$
\begin{gathered}
\mathcal{F}_{1}(A)= \begin{cases}1, & \text { if } A \in\{\phi, X\}, \\
1 / 2, & \text { if } A=\{a\}, \\
1 / 5, & \text { if } A=\{b\},\end{cases} \\
\mathcal{F}_{2}(A)= \begin{cases}1, & \text { if } A \in\{\phi, X\}, \\
1 / 8, & \text { if } A=\{a\}, \\
1 / 4, & \text { if } A=\{b\} .\end{cases}
\end{gathered}
$$

Note that $\left[\forall x\left(\{x\} \in \mathcal{F}_{1}\right)\right]=1 / 5 \not \leq 1 / 8=\left[\left(X, \tau_{1}, \tau_{2}\right) \in\right.$ $\left.T_{1}^{(i, j)}\right]$.

Example 3.3. Let $X=\{a, b\}$ and $\tau_{1}, \tau_{2}$ be two fuzzifying topologies defined as follows:

$$
\begin{gathered}
\tau_{1}(A)= \begin{cases}1, & \text { if } A \in\{\phi, X\}, \\
1 / 6, & \text { if } A=\{a\}, \\
1 / 3, & \text { if } A=\{b\},\end{cases} \\
\tau_{2}(A)= \begin{cases}1, & \text { if } A \in\{\phi, X\}, \\
1 / 4, & \text { if } A=\{a\}, \\
1 / 2, & \text { if } A=\{b\} .\end{cases}
\end{gathered}
$$

Note that

$$
\begin{gathered}
\mathcal{F}_{1}(A)= \begin{cases}1, & \text { if } A \in\{\phi, X\}, \\
1 / 3, & \text { if } A=\{a\}, \\
1 / 6, & \text { if } A=\{b\},\end{cases} \\
\mathcal{F}_{2}(A)= \begin{cases}1, & \text { if } A \in\{\phi, X\}, \\
1 / 2, & \text { if } A=\{a\}, \\
1 / 4, & \text { if } A=\{b\} .\end{cases}
\end{gathered}
$$

Note that $\left[\forall x\left(\{x\} \in \mathcal{F}_{2}\right)\right]=1 / 4 \not \leq 1 / 6=\left[\left(X, \tau_{1}, \tau_{2}\right) \in\right.$ $\left.T_{1}^{(i, j)}\right]$.

Theorem 3.5. Let $\left(X, \tau_{1}, \tau_{2}\right)$ be a fuzzifying bitopological 
space. Then

$$
\models\left(X, \tau_{1}, \tau_{2}\right) \in T_{1}^{(i, j)} \leftrightarrow \forall x\left(\{x\} \in \mathcal{F}^{p}\right) .
$$

Proof. For any $x_{1}, x_{2} \in X$ with $x_{1} \neq x_{2}$.

$$
\begin{aligned}
{\left[\forall x\left(\{x\} \in \mathcal{F}^{p}\right)\right] } & =\inf _{x \in X}\left[\{x\} \in \mathcal{F}^{p}\right] \\
& =\inf _{x \in X} \min \left(\left[\{x\} \in \mathcal{F}_{i}\right],\left[\{x\} \in \mathcal{F}_{j}\right]\right) \\
& \leq \inf _{x \in X}\left[\{x\} \in \mathcal{F}_{i}\right]=\inf _{x \in X} \tau_{i}(X \sim\{x\}) \\
& =\inf _{x \in X} \inf _{y \in X \sim\{x\}} N_{y}^{i}(X \sim\{x\}) \\
& \leq \inf _{y \in X \sim\left\{x_{2}\right\}} N_{y}^{i}\left(X \sim\left\{x_{2}\right\}\right) \\
& \leq N_{x_{1}}^{i}\left(X \sim\left\{x_{2}\right\}\right)=\sup _{x_{2} \notin A} N_{x_{1}}^{i}(A) .
\end{aligned}
$$

By the same way, we have

$$
\left[\forall x\left(\{x\} \in \mathcal{F}^{p}\right)\right] \leq \sup _{x_{1} \notin B} N_{x_{2}}^{j}(B) .
$$

So

$$
\begin{aligned}
{\left[\forall x\left(\{x\} \in \mathcal{F}^{p}\right)\right] } & \leq \inf _{x_{1} \neq x_{2}} \min \left(\sup _{x_{2} \notin A} N_{x_{1}}^{i}(A), \sup _{x_{1} \notin B} N_{x_{2}}^{j}(B)\right) \\
& =T_{1}^{(i, j)}\left(X, \tau_{1}, \tau_{2}\right) .
\end{aligned}
$$

On the other hand, from Theorem 3.4 we have

$$
\begin{aligned}
T_{1}^{(i, j)}\left(X, \tau_{1}, \tau_{2}\right) & \leq \inf _{x \in X} \min \left(\mathcal{F}_{i}(\{x\}), \mathcal{F}_{j}(\{x\})\right) \\
& =\left[\forall x\left(\{x\} \in \mathcal{F}^{p}\right)\right] .
\end{aligned}
$$

Therefore, $T_{1}^{(i, j)}\left(X, \tau_{1}, \tau_{2}\right)=\left[\forall x\left(\{x\} \in \mathcal{F}^{p}\right)\right]$.

Definition 3.5. Let $\left(X, \tau_{1}, \tau_{2}\right)$ be a fuzzifying bitopological space, we define

(1) ${ }^{1} T_{R_{i}}^{(i, j)}\left(X, \tau_{1}, \tau_{2}\right):=\forall x \forall U\left(x \in X \wedge U \in \mathcal{F}_{i} \wedge x \notin\right.$ $\left.U \rightarrow \exists A\left(A \in N_{x}^{i} \wedge c l_{j}(A) \cap U=\phi\right)\right)$.

(2) ${ }^{1} T_{R_{j}}^{(i, j)}\left(X, \tau_{1}, \tau_{2}\right):=\forall x \forall U\left(x \in X \wedge U \in \mathcal{F}_{j} \wedge x \notin\right.$ $\left.U \rightarrow \exists A\left(A \in N_{x}^{j} \wedge c l_{i}(A) \cap U=\phi\right)\right)$.

(3) ${ }^{1} T_{R}^{(i, j)}:={ }^{1} T_{R_{i}}^{(i, j)} \wedge{ }^{1} T_{R_{j}}^{(i, j)}$.

(4) ${ }^{2} T_{R_{i}}^{(i, j)}\left(X, \tau_{1}, \tau_{2}\right):=\forall x \forall V\left(x \in X \wedge V \in \tau_{i} \wedge x \in\right.$ $\left.V \rightarrow \exists B\left(B \in N_{x}^{i} \wedge c l_{j}(B) \subseteq V\right)\right)$.

(5) ${ }^{2} T_{R_{j}}^{(i, j)}\left(X, \tau_{1}, \tau_{2}\right):=\forall x \forall V\left(x \in X \wedge V \in \tau_{j} \wedge x \in\right.$ $\left.V \rightarrow \exists B\left(B \in N_{x}^{j} \wedge c l_{i}(B) \subseteq V\right)\right)$.

(6) ${ }^{2} T_{R}^{(i, j)}:={ }^{2} T_{R_{i}}^{(i, j)} \wedge{ }^{2} T_{R_{j}}^{(i, j)}$.
Theorem 3.6. Let $\left(X, \tau_{1}, \tau_{2}\right)$ be a fuzzifying bitopological space. Then
(1) $\models T_{R_{i}}^{(i, j)}\left(X, \tau_{1}, \tau_{2}\right) \leftrightarrow{ }^{n} T_{R_{i}}^{(i, j)}\left(X, \tau_{1}, \tau_{2}\right), n=1,2$.
(2) $\models T_{R_{j}}^{(i, j)}\left(X, \tau_{1}, \tau_{2}\right) \leftrightarrow{ }^{n} T_{R_{j}}^{(i, j)}\left(X, \tau_{1}, \tau_{2}\right), n=1,2$.
(3) $\models T_{R}^{(i, j)}\left(X, \tau_{1}, \tau_{2}\right) \leftrightarrow{ }^{n} T_{R}^{(i, j)}\left(X, \tau_{1}, \tau_{2}\right), n=1,2$.

Proof. It is similar to proof of Theorem 2.6 in [5].

Theorem 3.7. Let $\left(X, \tau_{1}, \tau_{2}\right)$ be a fuzzifying bitopological space, and let

(1) ${ }^{1} T_{N}^{(i, j)}\left(X, \tau_{1}, \tau_{2}\right):=\forall A \forall B\left(A \in \tau_{i} \wedge B \in \mathcal{F}_{j} \wedge B \subseteq\right.$ $\left.A \rightarrow \exists U \exists V\left(U \in \tau_{i} \wedge V \in \mathcal{F}_{j} \wedge B \subseteq U \subseteq V \subseteq A\right)\right)$.

(2) ${ }^{2} T_{N}^{(i, j)}\left(X, \tau_{1}, \tau_{2}\right):=\forall A \forall B\left(A \in \mathcal{F}_{i} \wedge B \in \mathcal{F}_{j} \wedge A \cap\right.$ $\left.B=\phi \rightarrow \exists U\left(U \in \tau_{i} \wedge A \subseteq U \wedge \operatorname{cl}_{j}(U) \cap B=\phi\right)\right)$.

(3) ${ }^{3} T_{N}^{(i, j)}\left(X, \tau_{1}, \tau_{2}\right):=\forall A \forall B\left(A \in \mathcal{F}_{i} \wedge B \in \tau_{j} \wedge A \subseteq\right.$ $\left.B \rightarrow \exists U\left(U \in \tau_{i} \wedge A \subseteq U \wedge c l_{j}(U) \subseteq B\right)\right)$.

Then $\models T_{N}^{(i, j)}\left(X, \tau_{1}, \tau_{2}\right) \leftrightarrow{ }^{n} T_{N}^{(i, j)}\left(X, \tau_{1}, \tau_{2}\right), n=1,2,3$.

Proof.

(1) $T_{N}^{(i, j)}\left(X, \tau_{1}, \tau_{2}\right)$

$=\inf _{A \cap B=\phi} \min \left(1,1-\min \left(\mathcal{F}_{i}(A), \mathcal{F}_{j}(B)\right)\right.$ $\left.+\sup _{U \cap V=\phi, A \subseteq V, B \subseteq U} \min \left(\tau_{i}(U), \tau_{j}(V)\right)\right)$

$=\inf _{X \sim A \cap B=\phi} \min \left(1,1-\min \left(\mathcal{F}_{i}(X \sim A), \mathcal{F}_{j}(B)\right)\right.$ $\left.+\sup _{U \cap X \sim V=\phi, X \sim A \subseteq X \sim V, B \subseteq U} \min \left(\tau_{i}(U), \tau_{j}(X \sim V)\right)\right)$

$=\inf _{B \subseteq A} \min \left(1,1-\min \left(\tau_{i}(A), \mathcal{F}_{j}(B)\right)\right.$

$$
\left.+\sup _{U \subseteq V, V \subseteq A, B \subseteq U} \min \left(\tau_{i}(U), \mathcal{F}_{j}(V)\right)\right)
$$$$
=\inf _{B \subseteq A} \min \left(1,1-\min \left(\tau_{i}(A), \mathcal{F}_{j}(B)\right)\right.
$$$$
\left.+\sup _{B \subseteq U \subseteq V \subseteq A} \min \left(\tau_{i}(U), \mathcal{F}_{j}(V)\right)\right)={ }^{1} T_{N}^{(i, j)}\left(X, \tau_{1}, \tau_{2}\right) .
$$

(2) and (3) are similar to that of Theorem 3.6

Now we define a new weaker form of pairwise normality in the fuzzifying bitopological spaces.

Definition 3.6. Let $\Omega$ be the class of all fuzzifying bitopological spaces. The unary fuzzy predicates ${ }^{w} T_{N}^{(i, j)} \in \Im(\Omega)$ defined as follows:

$$
\begin{aligned}
& \left(X, \tau_{1}, \tau_{2}\right) \in{ }^{w} T_{N}^{(i, j)} \\
& :=\forall A \forall B\left(A \in \mathcal{F}^{p} \wedge B \in \mathcal{F}^{p} \wedge A \cap B=\phi \rightarrow W_{A, B}^{(i, j)}\right) .
\end{aligned}
$$


Theorem 3.8. Let $\left(X, \tau_{1}, \tau_{2}\right)$ be a fuzzifying bitopological space. Then

$$
\models\left(X, \tau_{1}, \tau_{2}\right) \in T_{N}^{(i, j)} \rightarrow\left(X, \tau_{1}, \tau_{2}\right) \in{ }^{w} T_{N}^{(i, j)} .
$$

Proof. It is obtained from part (2) of Lemma 3.2 .

The following example shows that generally the reverse of Theorem 3.8 need not be true.

Example 3.4. Let $X=\{a, b, c, d\}, \tau_{1}, \tau_{2}$ be two fuzzifying topologies defined as follows:

$$
\begin{aligned}
& \tau_{1}(A)= \begin{cases}1, & \text { if } A \in\{\phi, X,\{a, b, d\}\}, \\
1 / 2, & \text { if } A=\{c\}, \\
0, & \text { if } o . w .,\end{cases} \\
& \tau_{2}(A)=\left\{\begin{array}{lll}
1, & \text { if } A \in\{\phi, X,\{b, c, d\}\}, \\
1 / 4, & \text { if } A=\{a\}, \\
0, & \text { if } \text { o.w.. }
\end{array}\right.
\end{aligned}
$$

Note that

$$
\begin{aligned}
& \mathcal{O}^{p}(A)= \begin{cases}1, & \text { if } A \in\{\phi, X\} \\
0, & \text { if } o . w .\end{cases}
\end{aligned}
$$

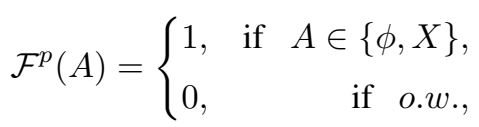

and

$$
\begin{aligned}
& \mathcal{F}_{1}(A)= \begin{cases}1, & \text { if } A \in\{\phi, X,\{c\}\}, \\
1 / 2, & \text { if } A=\{a, b, d\}, \\
0, & \text { if } o . w .,\end{cases} \\
& \mathcal{F}_{2}(A)=\left\{\begin{array}{lll}
1, & \text { if } A \in\{\phi, X,\{a\}\}, \\
1 / 4, & \text { if } A=\{b, c, d\}, \\
0, & \text { if } o . w . .
\end{array}\right.
\end{aligned}
$$

Then we have $\left[\left(X, \tau_{1}, \tau_{2}\right) \in{ }^{w} T_{N}^{(1,2)}\right]=1 \not \leq 0=\left[\left(X, \tau_{1}, \tau_{2}\right) \in\right.$ $\left.T_{N}^{(1,2)}\right]$.

\section{Relation among Separation Axioms in Fuzzi- fying Bitopological Spaces}

Theorem 4.1. Let $\left(X, \tau_{1}, \tau_{2}\right)$ be a fuzzifying bitopological space. Then

$$
\begin{aligned}
& \text { (1) } \models T_{R_{i}}^{(i, j)}\left(X, \tau_{1}, \tau_{2}\right) \dot{\wedge} T_{1}^{(i, j)}\left(X, \tau_{1}, \tau_{2}\right) \rightarrow T_{2}^{(i, j)}\left(X, \tau_{1}, \tau_{2}\right) . \\
& \text { (2) } \models T_{R_{j}}^{(i, j)}\left(X, \tau_{1}, \tau_{2}\right) \dot{\wedge} T_{1}^{(i, j)}\left(X, \tau_{1}, \tau_{2}\right) \rightarrow T_{2}^{(i, j)}\left(X, \tau_{1}, \tau_{2}\right) .
\end{aligned}
$$

Proof. From Theorem 3.5, the proof becomes obvious.

Theorem 4.2. Let $\left(X, \tau_{1}, \tau_{2}\right)$ be a fuzzifying bitopological space. Then

$$
\begin{aligned}
& (1) \models T_{4}^{(i, j)}\left(X, \tau_{1}, \tau_{2}\right) \rightarrow T_{R_{i}}^{(i, j)}\left(X, \tau_{1}, \tau_{2}\right) . \\
& (2) \models T_{4}^{(i, j)}\left(X, \tau_{1}, \tau_{2}\right) \rightarrow T_{R_{j}}^{(i, j)}\left(X, \tau_{1}, \tau_{2}\right) .
\end{aligned}
$$

Proof.

(1) Since $T_{4}^{(i, j)}\left(X, \tau_{1}, \tau_{2}\right)=\max \left(0, T_{N}^{(i, j)}\left(X, \tau_{1}, \tau_{2}\right)+\right.$ $\left.T_{1}^{(i, j)}\left(X, \tau_{1}, \tau_{2}\right)-1\right)$, then we prove that

$$
T_{R_{i}}^{(i, j)}\left(X, \tau_{1}, \tau_{2}\right) \geq T_{N}^{(i, j)}\left(X, \tau_{1}, \tau_{2}\right)+T_{1}^{(i, j)}\left(X, \tau_{1}, \tau_{2}\right)-1
$$

In fact,

$$
\begin{aligned}
& T_{N}^{(i, j)}\left(X, \tau_{1}, \tau_{2}\right)+T_{1}^{(i, j)}\left(X, \tau_{1}, \tau_{2}\right) \\
& =\inf _{U \cap V=\phi} \min \left(1,1-\min \left(\tau_{i}(X \sim U), \tau_{j}(X \sim V)\right)\right. \\
& \left.+\sup _{A \cap B=\phi, U \subseteq B, V \subseteq A} \min \left(\tau_{i}(A), \tau_{j}(B)\right)\right) \\
& +\inf _{z \in X} \min \left(\tau_{i}(X \sim\{z\}), \tau_{j}(X \sim\{z\})\right) \\
& \leq \inf _{x \notin U} \min \left(1,1-\min \left(\tau_{i}(X \sim U), \tau_{j}(X \sim\{x\})\right)\right. \\
& \left.+\sup _{A \cap B=\phi, U \subseteq B} \min \left(N_{x}^{i}(A), \tau_{j}(B)\right)\right) \\
& +\inf _{z \in X} \tau_{j}(X \sim\{z\}) \\
& =\inf _{x \notin U} \min \left(1, \max \left(1-\tau_{i}(X \sim U)\right.\right. \\
& +\sup _{A \cap B=\phi, U \subseteq B} \min \left(N_{x}^{i}(A), \tau_{j}(B)\right), 1-\tau_{j}(X \sim\{x\}) \\
& \left.+\sup _{A \cap B=\phi, U \subseteq B} \min \left(N_{x}^{i}(A), \tau_{j}(B)\right)\right) \\
& +\inf _{z \in X} \tau_{j}(X \sim\{z\}) \\
& =\inf _{x \notin U} \max \left(\operatorname { m i n } \left(1,1-\tau_{i}(X \sim U)\right.\right. \\
& \left.+\sup _{A \cap B=\phi, U \subseteq B} \min \left(N_{x}^{i}(A), \tau_{j}(B)\right)\right), \\
& \min \left(1,1-\tau_{j}(X \sim\{x\})\right. \\
& \left.\left.+\sup _{A \cap B=\phi, U \subseteq B} \min \left(N_{x}^{i}(A), \tau_{j}(B)\right)\right)\right) \\
& +\inf _{z \in X} \tau_{j}(X \sim\{z\}) \\
& \leq \inf _{x \notin U} \max \left(\operatorname { m i n } \left(1,1-\tau_{i}(X \sim U)\right.\right. \\
& \left.+\sup _{A \cap B=\phi, U \subseteq B} \min \left(N_{x}^{i}(A), \tau_{j}(B)\right)\right)+\tau_{j}(X \sim\{x\}), \\
& \min \left(1,1-\tau_{j}(X \sim\{x\})\right.
\end{aligned}
$$




$$
\begin{aligned}
& \left.\left.+\sup _{A \cap B=\phi, U \subseteq B} \min \left(N_{x}^{i}(A), \tau_{j}(B)\right)\right)+\tau_{j}(X \sim\{x\})\right) \\
\leq & \inf _{x \notin U} \max \left(\operatorname { m i n } \left(1,1-\tau_{i}(X \sim U)\right.\right. \\
& \left.+\sup _{A \cap B=\phi, U \subseteq B} \min \left(N_{x}^{i}(A), \tau_{j}(B)\right)\right)+\tau_{j}(X \sim\{x\}), \\
& \left.1+\sup _{A \cap B=\phi, U \subseteq B} \min \left(N_{x}^{i}(A), \tau_{j}(B)\right)\right) \\
\leq & \inf _{x \notin U} \min \left(1,1-\tau_{i}(X \sim U)\right. \\
& \left.+\sup _{A \cap B=\phi, U \subseteq B} \min \left(N_{x}^{i}(A), \tau_{j}(B)\right)\right)+1 \\
= & T_{R_{i}}^{(i, j)}\left(X, \tau_{1}, \tau_{2}\right)+1 .
\end{aligned}
$$

(2) It is similar to (1) above.

From Theorem 4.1 and Theorem 4.2 , we have the following result:

Corollary 4.1. Let $\left(X, \tau_{1}, \tau_{2}\right)$ be a fuzzifying bitopological space. Then

$$
\begin{aligned}
& (1) \models T_{3}^{(i, j)}\left(X, \tau_{1}, \tau_{2}\right) \rightarrow T_{2}^{(i, j)}\left(X, \tau_{1}, \tau_{2}\right) . \\
& (2) \models T_{4}^{(i, j)}\left(X, \tau_{1}, \tau_{2}\right) \rightarrow T_{R}^{(i, j)}\left(X, \tau_{1}, \tau_{2}\right) .
\end{aligned}
$$

The following example shows that generally $T_{4}^{(i, j)}\left(X, \tau_{1}, \tau_{2}\right) \rightarrow T_{3}^{(i, j)}\left(X, \tau_{1}, \tau_{2}\right)$ need not be true.

Example 4.1. For $X=\{a, b\}$, let $\tau_{1}$ and $\tau_{1}$ be two fuzzifying topologies, which are defined on $X$ in Example 3.2, then we have $T_{4}^{(i, j)}\left(X, \tau_{1}, \tau_{2}\right)=1 / 8 \not \leq 0=T_{3}^{(i, j)}\left(X, \tau_{1}, \tau_{2}\right)$.

Theorem 4.3. Let $\left(X, \tau_{1}, \tau_{2}\right)$ be a fuzzifying bitopological space. Then

$$
(1) \models\left(X, \tau_{1}, \tau_{2}\right) \in T_{1}^{(i, j)} \rightarrow\left(X, \tau_{1}, \tau_{2}\right) \in R_{0}^{(i, j)} \wedge
$$$$
\left(X, \tau_{1}, \tau_{2}\right) \in T_{0}^{(i, j)} \text {. }
$$

(2) If $T_{0}^{(i, j)}\left(X, \tau_{1}, \tau_{2}\right)=1$, then

$$
\begin{aligned}
\models & \left(X, \tau_{1}, \tau_{2}\right) \in T_{1}^{(i, j)} \leftrightarrow\left(X, \tau_{1}, \tau_{2}\right) \in R_{0}^{(i, j)} \\
& \wedge\left(X, \tau_{1}, \tau_{2}\right) \in T_{0}^{(i, j)} .
\end{aligned}
$$

\section{Proof.}

(1) It is obtained from part (1) of the Theorem 3.1 and part (2) Theorem 3.2 .

(2) Since $T_{0}^{(i, j)}\left(X, \tau_{1}, \tau_{2}\right)=1$, then for every $x, y \in X$ such that $x \neq y$, we have $\left[K_{x, y}^{(i, j)}\right]=1$. So

$$
\begin{aligned}
& R_{0}^{(i, j)}\left(X, \tau_{1}, \tau_{2}\right) \wedge T_{0}^{(i, j)}\left(X, \tau_{1}, \tau_{2}\right) \\
& =R_{0}^{(i, j)}\left(X, \tau_{1}, \tau_{2}\right)
\end{aligned}
$$

$$
\begin{aligned}
& =\inf _{x \neq y} \min \left(1,1-\left[K_{x, y}^{(i, j)}\right]+\left[H_{x, y}^{(i, j)}\right]\right) \\
& =\inf _{x \neq y}\left[H_{x, y}^{(i, j)}\right]=T_{1}^{(i, j)}\left(X, \tau_{1}, \tau_{2}\right) .
\end{aligned}
$$

Theorem 4.4. Let $\left(X, \tau_{1}, \tau_{2}\right)$ be a fuzzifying bitopological space. Then

$$
\begin{aligned}
& (1) \models\left(X, \tau_{1}, \tau_{2}\right) \in T_{2}^{(i, j)} \rightarrow\left(X, \tau_{1}, \tau_{2}\right) \in R_{1}^{(i, j)} \wedge \\
& \left(X, \tau_{1}, \tau_{2}\right) \in T_{0}^{(i, j)} . \\
& \text { (2) If } T_{0}^{(i, j)}\left(X, \tau_{1}, \tau_{2}\right)=1 \text {, then } \\
& \models\left(X, \tau_{1}, \tau_{2}\right) \in T_{2}^{(i, j)} \leftrightarrow\left(X, \tau_{1}, \tau_{2}\right) \in R_{1}^{(i, j)} \\
& \wedge\left(X, \tau_{1}, \tau_{2}\right) \in T_{0}^{(i, j)} .
\end{aligned}
$$

Proof.

(1) It is obtained from part (3) of the Theorem 3.1 and part (4) of the Theorem 3.2 .

(2) It is similar to proof of part (2) of the Theorem 4.3

Remark 4.1. In the crisp setting, i.e, if the underlying fuzzifying bitopology is the ordinary bitopology one can have that

$$
\begin{aligned}
& (1) \models\left(X, \tau_{1}, \tau_{2}\right) \in T_{1}^{(i, j)} \leftrightarrow\left(X, \tau_{1}, \tau_{2}\right) \in R_{0}^{(i, j)} \\
\wedge & \left(X, \tau_{1}, \tau_{2}\right) \in T_{0}^{(i, j)} . \\
& (2) \models\left(X, \tau_{1}, \tau_{2}\right) \in T_{2}^{(i, j)} \leftrightarrow\left(X, \tau_{1}, \tau_{2}\right) \in R_{1}^{(i, j)} \\
\wedge & \left(X, \tau_{1}, \tau_{2}\right) \in T_{0}^{(i, j)} .
\end{aligned}
$$

Generally these statements may not be true in fuzzifying bitopology as illustrated by the following example.

Example 4.2. Let $X=\{a, b\}$ and $\tau_{1}, \tau_{2}$ be two fuzzifying topologies defined as follows:

$$
\begin{gathered}
\tau_{1}(A)= \begin{cases}1, & \text { if } A \in\{\phi, X\}, \\
1 / 4, & \text { if } A=\{a\}, \\
1 / 5, & \text { if } A=\{b\},\end{cases} \\
\tau_{2}(A)=\left\{\begin{array}{lll}
1, & \text { if } A \in\{\phi, X\}, \\
1 / 3, & \text { if } A=\{a\}, \\
1 / 2, & \text { if } A=\{b\} .
\end{array}\right.
\end{gathered}
$$

Note that $T_{0}^{(i, j)}\left(X, \tau_{1}, \tau_{2}\right)=1 / 3, T_{1}^{(i, j)}\left(X, \tau_{1}, \tau_{2}\right)=1 / 5=$ $T_{2}^{(i, j)}\left(X, \tau_{1}, \tau_{2}\right)$ and $R_{0}^{(i, j)}\left(X, \tau_{1}, \tau_{2}\right)=3 / 4=R_{1}^{(i, j)}\left(X, \tau_{1}\right.$, $\left.\tau_{2}\right)$. Hence,

$$
\begin{aligned}
& R_{0}^{(i, j)}\left(X, \tau_{1}, \tau_{2}\right) \wedge T_{0}^{(i, j)}\left(X, \tau_{1}, \tau_{2}\right)=1 / 3 \neq 1 / 5 \\
& =T_{1}^{(i, j)}\left(X, \tau_{1}, \tau_{2}\right), \\
& R_{1}^{(i, j)}\left(X, \tau_{1}, \tau_{2}\right) \wedge T_{0}^{(i, j)}\left(X, \tau_{1}, \tau_{2}\right)=1 / 3 \neq 1 / 5
\end{aligned}
$$




$$
=T_{2}^{(i, j)}\left(X, \tau_{1}, \tau_{2}\right)
$$

Theorem 4.5. Let $\left(X, \tau_{1}, \tau_{2}\right)$ be a fuzzifying bitopological space. Then $T_{1}^{(i, j)}$

(1) $\left(X, \tau_{1}, \tau_{2}\right) \in R_{0}^{(i, j)} \dot{\wedge}\left(X, \tau_{1}, \tau_{2}\right) \in T_{0}^{(i, j)} \rightarrow\left(X, \tau_{1}, \tau_{2}\right) \in$

(2) If $T_{0}^{(i, j)}\left(X, \tau_{1}, \tau_{2}\right)=1$, then

$\models\left(X, \tau_{1}, \tau_{2}\right) \in R_{0}^{(i, j)} \dot{\wedge}\left(X, \tau_{1}, \tau_{2}\right) \in T_{0}^{(i, j)} \leftrightarrow\left(X, \tau_{1}, \tau_{2}\right)$

$\in T_{1}^{(i, j)}$.

\section{Proof.}

(1) It is clear.

(2) It is similar to the proof of part (2) of the Theorem 4.3

Theorem 4.6. Let $\left(X, \tau_{1}, \tau_{2}\right)$ be a fuzzifying bitopological space. Then $T_{2}^{(i, j)}$

$(1) \models\left(X, \tau_{1}, \tau_{2}\right) \in R_{1}^{(i, j)} \dot{\wedge}\left(X, \tau_{1}, \tau_{2}\right) \in T_{0}^{(i, j)} \rightarrow\left(X, \tau_{1}, \tau_{2}\right) \in$

(2) If $T_{0}^{(i, j)}\left(X, \tau_{1}, \tau_{2}\right)=1$, then

$$
\begin{aligned}
& \models\left(X, \tau_{1}, \tau_{2}\right) \in R_{1}^{(i, j)} \dot{\wedge}\left(X, \tau_{1}, \tau_{2}\right) \in T_{0}^{(i, j)} \\
& \leftrightarrow\left(X, \tau_{1}, \tau_{2}\right) \in T_{2}^{(i, j)} .
\end{aligned}
$$

Proof.

(1) It is clear.

(2) It is similar to the proof of part (2) of the Theorem 4.3

Theorem 4.7. Let $\left(X, \tau_{1}, \tau_{2}\right)$ be a fuzzifying bitopological space. Then

$(1) \models\left(X, \tau_{1}, \tau_{2}\right) \in T_{0}^{(i, j)} \rightarrow\left(\left(X, \tau_{1}, \tau_{2}\right) \in R_{0}^{(i, j)} \rightarrow\right.$ $\left.\left(X, \tau_{1}, \tau_{2}\right) \in T_{1}^{(i, j)}\right)$.

$(2) \models\left(X, \tau_{1}, \tau_{2}\right) \in R_{0}^{(i, j)} \rightarrow\left(\left(X, \tau_{1}, \tau_{2}\right) \in T_{0}^{(i, j)} \rightarrow\right.$ $\left.\left(X, \tau_{1}, \tau_{2}\right) \in T_{1}^{(i, j)}\right)$.

$(3) \models\left(X, \tau_{1}, \tau_{2}\right) \in T_{0}^{(i, j)} \rightarrow\left(\left(X, \tau_{1}, \tau_{2}\right) \in R_{1}^{(i, j)} \rightarrow\right.$ $\left.\left(X, \tau_{1}, \tau_{2}\right) \in T_{2}^{(i, j)}\right)$.

$(4) \models\left(X, \tau_{1}, \tau_{2}\right) \in R_{1}^{(i, j)} \rightarrow\left(\left(X, \tau_{1}, \tau_{2}\right) \in T_{0}^{(i, j)} \rightarrow\right.$ $\left.\left(X, \tau_{1}, \tau_{2}\right) \in T_{2}^{(i, j)}\right)$.

Proof. From part (1) of the Theorem 3.1. part (2) of the Theorem 3.2 and part (3) of the Theorem 4.3 , the proof becomes obvious.

\section{Separation Axioms and Continuity in Fuzzi- fying Bitopological Spaces}

Theorem 5.1. Let $\left(X, \tau_{1}, \tau_{2}\right)$ and $\left(Y, \sigma_{1}, \sigma_{2}\right)$ be two fuzzifying bitopological spaces. If a mapping $f:\left(X, \tau_{1}, \tau_{2}\right) \rightarrow$ $\left(Y, \sigma_{1}, \sigma_{2}\right)$ is injective and pairwise fuzzy open with degree one, then
$(1) \models\left(X, \tau_{1}, \tau_{2}\right) \in T_{0}^{(i, j)} \rightarrow\left(Y, \sigma_{1}, \sigma_{2}\right) \in T_{0}^{(i, j)}:$
(2) $\models\left(X, \tau_{1}, \tau_{2}\right) \in T_{1}^{(i, j)} \rightarrow\left(Y, \sigma_{1}, \sigma_{2}\right) \in T_{1}^{(i, j)}$;
(3) $\models\left(X, \tau_{1}, \tau_{2}\right) \in T_{2}^{(i, j)} \rightarrow\left(Y, \sigma_{1}, \sigma_{2}\right) \in T_{2}^{(i, j)}$.

Proof.

(1) From part (1) of the Theorem 3.2 in [8], $\left[f \in O_{1}\right]=1$ and $\left[f \in O_{2}\right]=1$, we have for every $v \in P(Y)$ and $x \in X, N_{x}^{t}\left(f^{-1}(V)\right) \leq N_{f(x)}^{t}(V), t=1,2$. Therefore,

$$
\begin{aligned}
T_{0}^{(i, j)}\left(X, \tau_{1}, \tau_{2}\right) \\
=\inf _{x \neq y} \max \left(\sup _{y \notin A} N_{x}^{i}(A), \sup _{x \notin A} N_{y}^{j}(A)\right) \\
=\inf _{x \neq y} \max \left(\sup _{y \notin A} N_{x}^{i}\left(f^{-1} f(A)\right), \sup _{x \notin A} N_{y}^{j}\left(f^{-1} f(A)\right)\right) \\
\leq \inf _{x \neq y} \max \left(\sup _{y \notin A} N_{f(x)}^{i}(f(A)), \sup _{x \notin A} N_{f(y)}^{j}(f(A))\right) \\
=\inf _{f(x) \neq f(y)} \max _{f(y) \notin f(A)} N_{f(x)}^{i}(f(A)), \\
\left.\quad \sup _{f(x) \notin f(A)} N_{f(y)}^{j}(f(A))\right) \\
=\inf _{z \neq w} \max \left(\sup _{w \notin H} N_{z}^{i}(H), \sup _{z \notin H} N_{w}^{j}(H)\right) \\
=T_{0}^{(i, j)}\left(Y, \sigma_{1}, \sigma_{2}\right) .
\end{aligned}
$$

The proof of (2) and (3) is similar to (1) above.

Theorem 5.2. Let $\left(X, \tau_{1}, \tau_{2}\right)$ and $\left(Y, \sigma_{1}, \sigma_{2}\right)$ be two fuzzifying bitopological spaces. If a mapping $f:\left(X, \tau_{1}, \tau_{2}\right) \rightarrow$ $\left(Y, \sigma_{1}, \sigma_{2}\right)$ is injective and pairwise fuzzy continuous with degree one, then
(1) $\models\left(Y, \sigma_{1}, \sigma_{2}\right) \in T_{0}^{(i, j)} \rightarrow\left(X, \tau_{1}, \tau_{2}\right) \in T_{0}^{(i, j)} ;$
(2) $\models\left(Y, \sigma_{1}, \sigma_{2}\right) \in T_{1}^{(i, j)} \rightarrow\left(X, \tau_{1}, \tau_{2}\right) \in T_{1}^{(i, j)}$;
(3) $\models\left(Y, \sigma_{1}, \sigma_{2}\right) \in T_{2}^{(i, j)} \rightarrow\left(X, \tau_{1}, \tau_{2}\right) \in T_{2}^{(i, j)}$.

Proof. From part (3) of the Theorem 2.1 in [4] the proof becomes obvious.

Theorem 5.3. Let $\left(X, \tau_{1}, \tau_{2}\right)$ and $\left(Y, \sigma_{1}, \sigma_{2}\right)$ be two fuzzifying bitopological spaces. If a mapping $f:\left(X, \tau_{1}, \tau_{2}\right) \rightarrow$ $\left(Y, \sigma_{1}, \sigma_{2}\right)$ is bijective, pairwise fuzzy open and pairwise fuzzy 
continuous with degree one, then

$$
\begin{aligned}
& (1) \models\left(X, \tau_{1}, \tau_{2}\right) \in T_{N}^{(i, j)} \leftrightarrow\left(Y, \sigma_{1}, \sigma_{2}\right) \in T_{N}^{(i, j)} ; \\
& (2) \models\left(X, \tau_{1}, \tau_{2}\right) \in T_{R}^{(i, j)} \leftrightarrow\left(Y, \sigma_{1}, \sigma_{2}\right) \in T_{R}^{(i, j)} .
\end{aligned}
$$

Proof.

(1) (a) Since $f$ is injective, pairwise open and pairwise continuous with degree one, we have

$$
\begin{aligned}
& {\left[T_{N}^{(i, j)}\left(X, \tau_{1}, \tau_{2}\right)\right]=\inf _{A \cap B=\phi} \min \left(1,1-\min \left(\mathcal{F}_{i}(A), \mathcal{F}_{j}(B)\right)\right.} \\
& \left.+\sup _{U \cap V=\phi, A \subseteq V, B \subseteq U} \min \left(\tau_{i}(U), \tau_{j}(V)\right)\right) \\
& =\inf _{A \cap B=\phi} \min \left(1,1-\min \left(\tau_{i}(X \sim A), \tau_{j}(X \sim B)\right)\right. \\
& \left.+\sup _{U \cap V=\phi, A \subseteq V, B \subseteq U} \min \left(\tau_{i}(U), \tau_{j}(V)\right)\right) \\
& \geq \inf _{A \cap B=\phi} \min \left(1,1-\min \left(\sigma_{i}(f(X \sim A)), \sigma_{j}(f(X \sim B))\right)\right. \\
& \left.+\sup _{U \cap V=\phi, A \subseteq V, B \subseteq U} \min \left(\tau_{i}\left(f^{-1} f(U)\right), \tau_{j}\left(f^{-1} f(V)\right)\right)\right) \\
& \geq \inf _{A \cap B=\phi} \min \left(1,1-\min \left(\sigma_{i}(Y \sim f(A)), \sigma_{j}(Y \sim f(B))\right)\right. \\
& \left.+\sup _{U \cap V=\phi, A \subseteq V, B \subseteq U} \min \left(\sigma_{i}(f(U)), \sigma_{j}(f(V))\right)\right) \\
& =\inf _{H \cap G=\phi} \min \left(1,1-\min \left(\sigma_{i}(Y \sim H), \sigma_{j}(Y \sim G)\right)\right. \\
& \left.+\sup _{M \cap N=\phi, H \subseteq N, G \subseteq M} \min \left(\sigma_{i}(M), \sigma_{j}(N)\right)\right) \\
& =\left[T_{N}^{(i, j)}\left(Y, \sigma_{1}, \sigma_{2}\right)\right] \text {. }
\end{aligned}
$$

(b) Since $f$ is surjective, pairwise open and pairwise continuous with degree one, we have

$$
\begin{aligned}
& {\left[T_{N}^{(i, j)}\left(Y, \sigma_{1}, \sigma_{2}\right)\right]} \\
& =\inf _{H \cap G=\phi} \min \left(1,1-\min \left(\sigma_{i}(Y \sim H), \sigma_{j}(Y \sim G)\right)\right. \\
& \left.\quad+\sup _{M \cap N=\phi, H \subseteq N, G \subseteq M} \min \left(\sigma_{i}(M), \sigma_{j}(N)\right)\right) \\
& \geq \inf _{H \cap G=\phi} \min \left(1,1-\min \left(\tau_{i}\left(f^{-1}(Y \sim H)\right),\right.\right. \\
& \left.\tau_{j}\left(f^{-1}(Y \sim G)\right)\right) \\
& \quad+\sup _{M \cap N=\phi, H \subseteq N, G \subseteq M} \min \left(\sigma_{i}\left(f f^{-1}(M)\right),\right. \\
& \left.\left.\sigma_{j}\left(f f^{-1}(N)\right)\right)\right) \\
& \geq \inf _{H \cap G=\phi} \min \left(1,1-\min \left(\tau_{i}\left(X \sim f^{-1}(H)\right),\right.\right. \\
& \left.\quad \tau_{j}\left(Y \sim f^{-1}(G)\right)\right) \\
& \left.\quad+\sup _{M \cap N=\phi, H \subseteq N, G \subseteq M} \min \left(\tau_{i}\left(f^{-1}(M)\right), \tau_{j}\left(f^{-1}(N)\right)\right)\right)
\end{aligned}
$$

$$
\begin{aligned}
= & \inf _{A \cap B=\phi} \min \left(1,1-\min \left(\tau_{i}(X \sim A), \tau_{j}(X \sim B)\right)\right. \\
& \left.+\sup _{U \cap V=\phi, A \subseteq V, B \subseteq U} \min \left(\tau_{i}(U), \tau_{j}(V)\right)\right) \\
= & {\left[T_{N}^{(i, j)}\left(X, \tau_{1}, \tau_{2}\right)\right] . }
\end{aligned}
$$

From (a) and (b), we have $\left[T_{N}^{(i, j)}\left(X, \tau_{1}, \tau_{2}\right)\right]=\left[T_{N}^{(i, j)}\left(Y, \sigma_{1}\right.\right.$, $\left.\left.\sigma_{2}\right)\right]$.

(2) It is similar to (1) above.

\section{Conclusions}

In the present paper we used Łukasiewicz fuzzy logic to extend the notions of separation axioms from the framework of fuzzifying topological spaces into the framework of fuzzifying bitopological spaces and study some relations between them Also we investigate the image of these kinds of fuzzifying bitopological spaces under some types of fuzzy mappings.

\section{Conflict of Interest}

No potential conflict of interest relevant to this article was reported.

\section{References}

[1] J. C. Kelly, "Bitopological spaces," Proceedings of the London Mathematical Society, vol. 3, no. 1, pp. 71-89, 1963. https://doi.org/10.1112/plms/s3-13.1.71

[2] M. Ying, "A new approach for fuzzy topology (I)," Fuzzy Sets and Systems, vol. 39, no. 3, pp. 303-321, 1991. https: //doi.org/10.1016/0165-0114(91)90100-5

[3] M. Ying, "A new approach for fuzzy topology (II)," Fuzzy Sets and Systems, vol. 47, no. 2, pp. 221-232, 1992. https: //doi.org/10.1016/0165-0114(92)90181-3

[4] M. Ying, "A new approach for fuzzy topology (III)," Fuzzy Sets and Systems, vol. 55, no. 2, pp. 193-207, 1993. https: //doi.org/10.1016/0165-0114(93)90132-2

[5] J. Shen, "Separation axiom in fuzzifying topology," Fuzzy Sets and Systems, vol. 57, no. 1, pp. 111-123, 1993. https: //doi.org/10.1016/0165-0114(93)90124-Z

[6] G. Zhang and M. Liu, "On properties of $\theta(i, j)$-open sets in fuzzifying bitopological space," Journal of Fuzzy Mathematics, vol. 11, no. 1, pp. 165-178, 2003. 
[7] A. Kilicman and Z. Salleh, "On pairwise Lindelöf bitopological spaces," Topology and its Applications, vol. 154, no. 8, pp. 1600-1607, 2007. https://doi.org/10.1016/j.topol. 2006.12.007

[8] A. A. Allam, A. M. Zahran, A. K. Mousa, and H. M. Binshahnah, "New types of continuity and openness in fuzzifying bitopological spaces," Journal of the Egyptian Mathematical Society, vol. 24, no. 2, pp. 286-294, 2016. https://doi.org/10.1016/j.joems.2015.05.005

[9] F. H. Khedr, F. M. Zeyada, and O. R. Sayed, "On separation axioms in fuzzifying topology," Fuzzy Sets and Systems, vol. 119, no. 3, pp. 439-458, 2001. https://doi.org/10.1016/ S0165-0114(99)00077-9

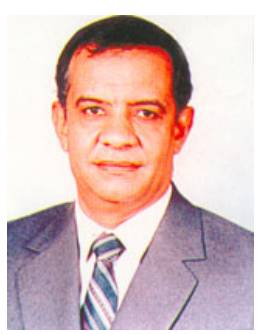

Ahmed Abd El-Monsef Allam s a professor of Department of Mathematics, Assiut University, Egypt. His research areas are fuzzy topology and general topology.

E-mail: allam51ahmed@yahoo.com

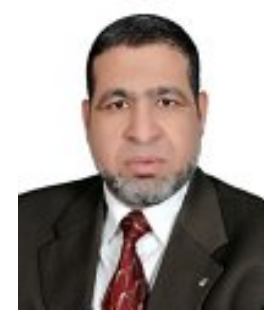

Ahmed Mohammed Zahran is a professor of Department of Mathematics, Al-Azhar University, Egypt. His research areas are fuzzy topology and general topology.

E-mail: amzahran@azhar.edu.eg

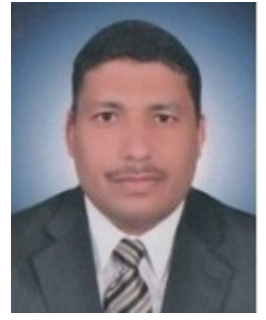

Ahmed Khalf Mousa is a associate professor of Department of Mathematics, AlAzhar University, Egypt. His research areas are fuzzy topology, general topology, and GIS.

E-mail: akmousa@azhar.edu.eg

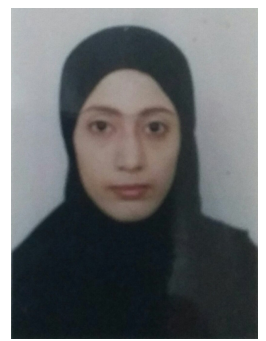

Hana Mohsen Binshahnah is a assistant professor of Department of Mathematics, Hadhramout University, Yemen. His research areas are fuzzy topology and general topology.

E-mail: hmbsh2006@yahoo.com 